ARTICLE

https://doi.org/10.1038/s41467-019-10515-3

\title{
Indirect chiral magnetic exchange through Dzyaloshinskii-Moriya-enhanced RKKY interactions in manganese oxide chains on $\operatorname{Ir}(100)$
}

\author{
Martin Schmitt ${ }^{1}$, Paolo Moras (10 2 ${ }^{2}$, Gustav Bihlmayer (10 ${ }^{3}$, Ryan Cotsakis ${ }^{1,4}$, Matthias Vogt ${ }^{1}$, \\ Jeannette Kemmer ${ }^{1,8}$, Abderrezak Belabbes ${ }^{5}$, Polina M. Sheverdyaeva ${ }^{2}$, Asish K. Kundu ${ }^{6}$, Carlo Carbone ${ }^{2}$, \\ Stefan Blügel (i) ${ }^{3} \&$ Matthias Bode (iD ${ }^{1,7}$
}

Localized electron spins can couple magnetically via the Ruderman-Kittel-Kasuya-Yosida interaction even if their wave functions lack direct overlap. Theory predicts that spin-orbit scattering leads to a Dzyaloshinskii-Moriya type enhancement of this indirect exchange interaction, giving rise to chiral exchange terms. Here we present a combined spin-polarized scanning tunneling microscopy, angle-resolved photoemission, and density functional theory study of $\mathrm{MnO}_{2}$ chains on $\operatorname{Ir}(100)$. Whereas we find antiferromagnetic $\mathrm{Mn}-\mathrm{Mn}$ coupling along the chain, the inter-chain coupling across the non-magnetic Ir substrate turns out to be chiral with a $120^{\circ}$ rotation between adjacent $\mathrm{MnO}_{2}$ chains. Calculations reveal that the Dzyaloshinskii-Moriya interaction results in spin spirals with a periodicity in agreement with experiment. Our findings confirm the existence of indirect chiral magnetic exchange, potentially giving rise to exotic phenomena, such as chiral spin-liquid states in spin ice systems or the emergence of new quasiparticles.

\footnotetext{
${ }^{1}$ Physikalisches Institut, Experimentelle Physik II, Universität Würzburg, Am Hubland, 97074 Würzburg, Germany. ${ }^{2}$ Istituto di Struttura della MateriaCNR (ISM-CNR), Trieste 34149, Italy. ${ }^{3}$ Peter Grünberg Institut and Institute for Advanced Simulation, Forschungszentrum Jülich \& JARA, 52425 Jülich, Germany. ${ }^{4}$ University of British Columbia, 2329 West Mall, Vancouver, BC V6T 1Z4, Canada. ${ }^{5}$ Physical Science and Engineering Division, King Abdullah University of Science \& Technology (KAUST), Thuwal 23955-6900, Saudi Arabia. ${ }^{6}$ International Center for Theoretical Physics (ICTP), Trieste 34151, Italy. ${ }^{7}$ Wilhelm Conrad Röntgen-Center for Complex Material Systems (RCCM), Universität Würzburg, Am Hubland, 97074 Würzburg, Germany.

${ }^{8}$ Present address: California Institute of Technology, 1200 E. California Blvd., Pasadena, CA 91125, USA. Correspondence and requests for materials should be addressed to P.M. (email: paolo.moras@trieste.ism.cnr.it)
} 
T he concept of the Ruderman-Kittel-Kasuya-Yosida (RKKY) interaction ${ }^{1,2}$ has successfully been applied to explain the magnetic properties of numerous indirectly coupled material systems which cannot be properly described by direct Heisenberg exchange. Prominent examples are the rareearth metals with their partially filled but highly localized $4 f$ shell ${ }^{3,4}$ or magnetic multilayers separated by non-magnetic metallic spacers. Since spin-orbit-related effects play no significant role in conventional RKKY, practical realizations are largely limited to collinear coupling terms, where-depending on spacer thickness and Fermi wave length-the relative magnetic orientation is either parallel or antiparallel $l^{5}$. Nevertheless, the giant magneto-resistance effect of layered magnetic materials is widely used in spin valve applications for field sensors or magnetic read heads ${ }^{6}$.

Theory predicts that spin-orbit scattering leads to a Dzyaloshinskii-Moriya $^{7,8}$ type enhancement of the RKKY interaction $^{9,10}$, or DME-RKKY in short, giving rise to chiral exchange terms. First evidence of indirect chiral magnetic exchange in layered structures was obtained from magnetic fielddependent neutron diffraction studies Dy/Y superlattices ${ }^{11,12}$. Further experimental evidence of DME-RKKY is essentially limited to non-collinear spin structures observed in surfacedeposited clusters ${ }^{13-16}$.

Here we report on the direct observation of chiral magnetic order between $\mathrm{MnO}_{2}$ chains which is mediated by RKKY interaction via conduction electrons of the Ir substrate. The strong spin-orbit coupling in Ir leads to an appreciable DMI, resulting in a chiral spin spiral with a $120^{\circ}$ rotation between adjacent $\mathrm{MnO}_{2}$ chains.

\section{Results}

Structural and electronic properties. The growth and structural properties of self-organized transition metal oxide (TMO) chains on $\operatorname{Ir}(001)$ have recently been studied by means of STM and lowenergy electron diffraction (LEED) $)^{17}$. It has been shown that many TMOs form extended $(3 \times 1)$-ordered domains. Depending on the particular transition metal element, various intra-chain spin structures (along the chain) were predicted by DFT calculations ${ }^{17}$, ranging from a non-magnetic $\mathrm{NiO}_{2}$, over ferromagnetically ordered (FM) $\mathrm{CoO}_{2}$, to antiferromagnetic (AFM) $\mathrm{FeO}_{2}$ and $\mathrm{MnO}_{2}$ chains. In contrast, only a very weak inter-chain magnetic coupling between adjacent chains across the $\operatorname{Ir}(001)$ substrate was predicted $^{17}$, too weak to result in spontaneous, permanent, and long-range magnetic order.

A topographic STM image of a typical $\mathrm{MnO}_{2} / \operatorname{Ir}(001)$ surface is shown in Fig. 1a. Wide flat terraces are decorated by roughly rectangularly shaped islands of atomic height. Terraces and islands both exhibit stripes running along the [110] or the [110] direction. These stripes originate from the self-organized growth of $\mathrm{MnO}_{2}$ chains which leads to a $(3 \times 1)$ structural unit cell ${ }^{17}$. Some domain boundaries can be recognized which separate domains which differ either in stripe direction (left arrow in Fig. 1a) or by an incommensurate phase shift (right arrow). The higher resolution image of Fig. $1 \mathrm{~b}$ was measured on a single $(3 \times$ 1) domain. The stripe periodicity of $(840 \pm 50) \mathrm{pm}$, corresponds well to the expected value of $3 \times a_{\mathrm{Ir}}=816 \mathrm{pm}$, with the Ir lattice constant $a_{\mathrm{Ir}}=272 \mathrm{pm}^{17}$. Only 36 defects are observed (24 bright spots; 9 dumbbells, 2 point-like hole; 1 line defect), equivalent to a chain defect density below $0.35 \%$.

The structure of the $\mathrm{MnO}_{2}$ chains on $\operatorname{Ir}(001)$ as proposed by Ferstl et al. ${ }^{17}$ is schematically represented in Fig. 1c. Along the chains nearest-neighbor $\mathrm{Mn}$ atoms (yellow) are separated by two oxygen atoms (red). Interestingly, the $\mathrm{MnO}_{2}$ chains sit above empty substrate rows, held in place by the oxygens atoms. DFT
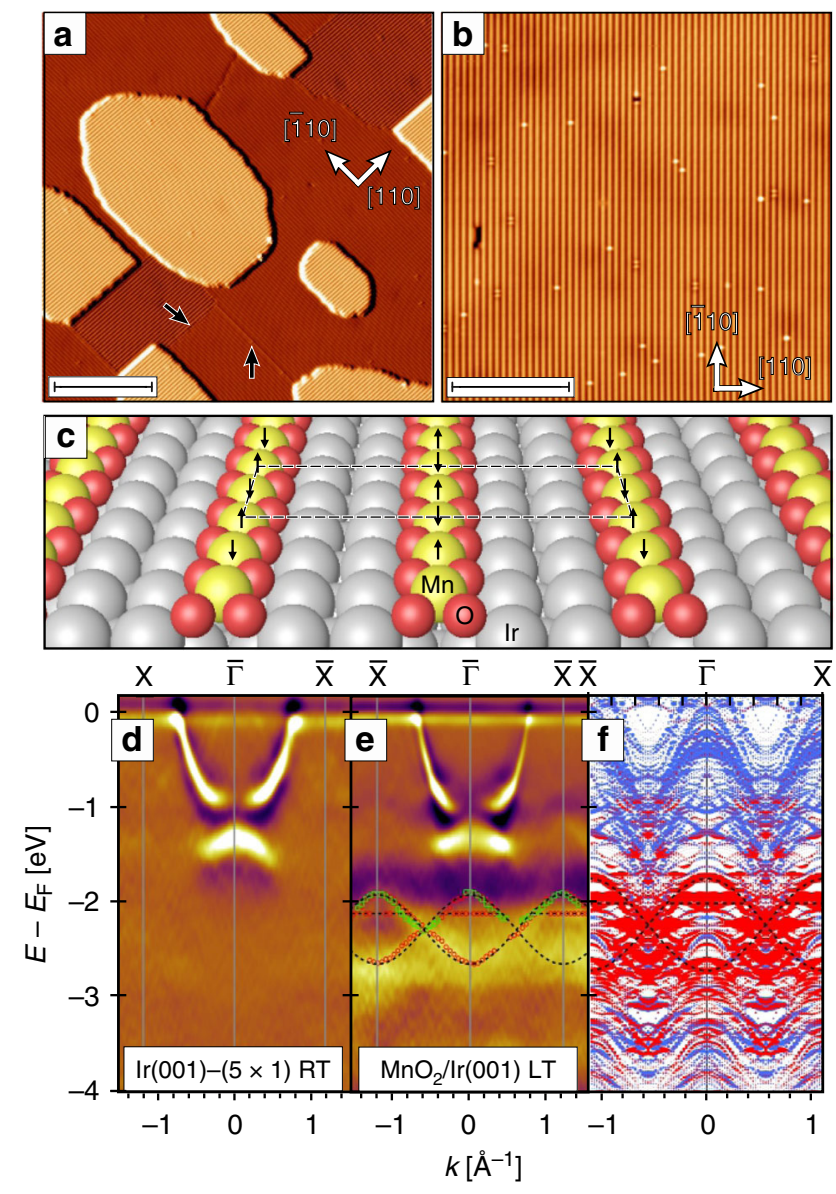

Fig. 1 Structural and electronic properties of $\mathrm{MnO}_{2}$ on $\operatorname{Ir}(001)$. a Large scale STM image showing islands of monolayer height (scale bar: $25 \mathrm{~nm}$ ). The entire surface including the islands are covered by chains along the [110] or [110] direction. b Higher resolution STM image of the $\mathrm{MnO}_{2}$ chains (scale bar: $15 \mathrm{~nm}$ ). Scan parameters: $U=1 \mathrm{~V}, I=300 \mathrm{pA}$. c Schematic model of the atomic structure and the AFM $(6 \times 2)$ spin arrangement predicted in ref. ${ }^{17}$. d, e Second derivative ARPES data of the $\operatorname{Ir}(001)-(5 \times 1)$ surface (at room temperature, $\mathrm{RT}$ ) and for $\mathrm{MnO}_{2} / \operatorname{Ir}(001)$ (at $13 \mathrm{~K}, \mathrm{LT}$ ) along the $\bar{\Gamma}-\bar{X}$ direction $(h \nu=150 \mathrm{eV})$. Red and green symbols mark the dispersion of $\mathrm{Mn}$ related states as determined from data taken at $h \nu=150 \mathrm{eV}$ and $130 \mathrm{eV}$, respectively. Their periodicity is in agreement with the $2 \times$ AFM order expected along the chains. Dashed lines are guides to the eye. $f$ DFT band structure of $\mathrm{MnO}_{2} / \mathrm{Ir}(001)$ exhibiting AFM order along the chains. Red and blue dots represent $\mathrm{Mn}$ and Ir states, respectively. The size of the symbols indicates the surface localization of the corresponding state. The same dashed lines shown in e (stretched by a factor 1.33 to consider the larger band width in DFT) agree well with surface-localized Mn states

calculations predicted an AFM coupling along the $\mathrm{MnO}_{2}$ chains, favored by $27 \mathrm{meV}$ per $\mathrm{Mn}$ pair with respect to a FM coupling ${ }^{17}$. Due to the large separation between adjacent chains a much weaker AFM coupling with an energy gain of $0.4 \mathrm{meV}$ per $\mathrm{Mn}$ pair was found across the stripes, overall resulting in a rectangular $(6 \times 2)$ magnetic unit cell, sketched in Fig. 1c. ARPES measurements support the presence of an AFM intra-chain coupling. Figure 1d, e display second derivative ARPES spectra along the $\bar{\Gamma}-\bar{X}$ axis of the $\operatorname{Ir}(001)$ surface Brillouin zone (SBZ) (corresponding to the $\operatorname{Ir}[110]$ direction) for the clean $(5 \times 1)$-reconstructed substrate and the $\mathrm{MnO}_{2} / \mathrm{Ir}(001)$ system, respectively. The second derivative is used to enhance the sensitivity to Mn-related states, which are broadened by the hybridization with the substrate. 
The photon energy is chosen such that the Ir $5 d$ signal is weak, except for some bulk bands dispersing symmetrically about $\bar{\Gamma}$ within $1.6 \mathrm{eV}$ below $E_{\mathrm{F}}$. Upon formation of the $\mathrm{MnO}_{2}$ chains new states appear between -1.9 and $-2.9 \mathrm{eV}$ (Fig. 1e). The peak positions of Mn-related states are marked by red $(h v=150 \mathrm{eV})$ and green symbols $(h v=130 \mathrm{eV})$. Two sinusoidal dashed lines having maxima and minima at the $\bar{\Gamma}$ and $\bar{X}$ points are guides to the eye connecting the dispersive states. Flat states below the maxima are connected by dashed segments (see Supplementary Note 1 for bare data). These lines are compared with first-principles electronic structure calculations of AFM $\mathrm{MnO}_{2}$ chains on $\operatorname{Ir}(001)$ oriented along the $y$-direction (Fig. 1f). They match well with the energy position of surfacelocalized Mn bands, but the experimental data display a smaller band width than DFT by a factor of 1.33, probably due to correlation effects. As detailed in the Supplementary Note 2 the sinusoidal bands mainly consist of $d_{y z}$ and $d_{x^{2}-y^{2}}$ states, whereas the flat band is dominated by states with $d_{z x}$ and $d_{z^{2}}$ character. This observation suggests the presence of a $2 \times$ periodicity, which turns the $\bar{X}$ points of the original SBZ into $\bar{\Gamma}$ points of the reduced SBZ, as expected for an AFM supercell. Other features located between -1 and $-1.5 \mathrm{eV}$ can be interpreted as surface umklapps of the Ir bulk band near $\bar{\Gamma}$ that repeat according to the AFM supercell. We recall here that the ARPES measurements of Fig. 1e average over both directions parallel and perpendicular to the chains, as a consequence of the domain structure of the system (Fig. 1a). The inter-chain coupling which results in a $9 \times$ magnetic unit cell (see below) is expected to be much weaker than the direct intra-chain AFM coupling and does not give rise to dispersive features in the ARPES data.

Spin-polarized scanning tunneling microscopy. Figure 2a shows an atomic scale STM image of $\mathrm{MnO}_{2}$ chains on $\operatorname{Ir}(001)$ taken with a non-magnetic $\mathrm{W}$ tip. The data show a structural $(3 \times 1)$ unit cell (black box) and nicely reproduce earlier measurements ${ }^{17}$. The black lines in Fig. 2c show line profiles taken along the three adjacent $\mathrm{MnO}_{2}$ chains indicated by arrows in Fig. 2a. They exhibit a periodicity $(287 \pm 20) \mathrm{pm}$, agreeing well with $a_{\mathrm{Ir}}$, i.e., the $\mathrm{Mn}-\mathrm{Mn}$ inter-atomic distance expected along the chain. Note, that within the noise level achievable in our setup the corrugation amplitude of $(1.9 \pm 0.1) \mathrm{pm}$ remains constant.

As we will describe in the following, our spin-polarized STM experiments exhibit some additional contrasts which allow to elucidate the spin structure of the $\mathrm{MnO}_{2}$ chains on $\operatorname{Ir}(001)$. Figure $2 \mathrm{~b}$ shows an SP-STM image scanned with an in-plane sensitive Cr-coated W tip. Comparison with the spin-averaged data presented in Fig. 2a reveals two qualitative differences: (i) The periodicity measured with magnetic tips along the chains is longer and (ii) the contrast observed on different $\mathrm{MnO}_{2}$ chains is not constant but becomes significantly smaller for every third chain. Again we analyzed line profiles taken along three adjacent $\mathrm{MnO}_{2}$ chains in between the colored arrows in Fig. 2b. These data, which are plotted in the bottom part of Fig. 2c, immediately illustrates a doubling of the periodicity, $2 a_{\mathrm{Ir}}$. This SP-STM contrast is characteristic for alternating spins ${ }^{18}$ and consistent with the proposed AFM Mn-Mn coupling along the chains ${ }^{17}$. Furthermore, the spin-polarized data reveal a systematic variation of the corrugation. For example, the blue line trace exhibits a corrugation of $6.1 \mathrm{pm}$, in contrast to $3.2 \mathrm{pm}$ (green) and $5.4 \mathrm{pm}$ (red) for the two adjacent $\mathrm{MnO}_{2}$ chains. Finally, there is also a distinct phase relation between the chains. Comparing the three colored traces plotted in Fig. $2 \mathrm{c}$ a $\pi$ phase shift between the blue and the green trace becomes apparent, whereas no phase shift occurs between the green and the red trace.

Modeling the magnetic contrast. The SP-STM contrast observed on $\mathrm{MnO}_{2}$ chains can semi-quantitatively be understood by
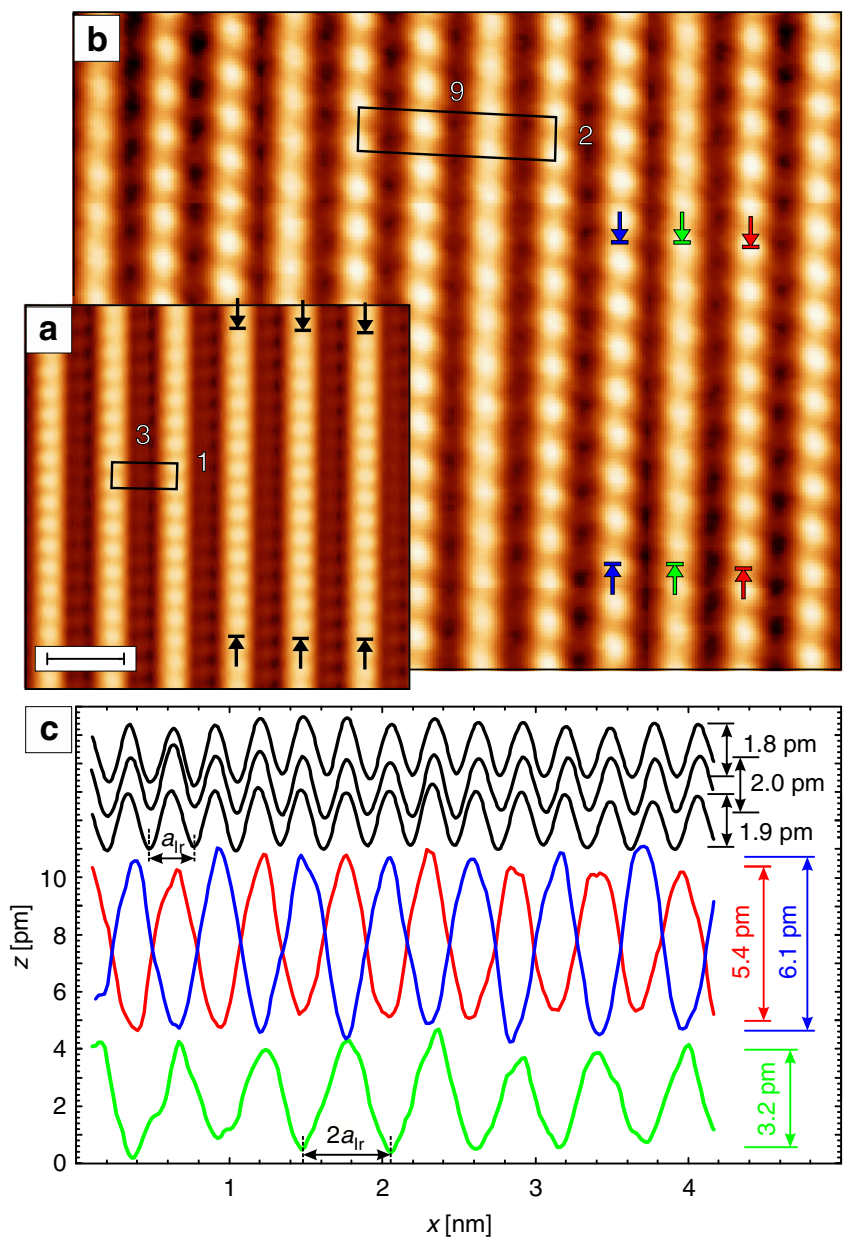

Fig. 2 Atomic resolution scans of $\mathrm{MnO}_{2}$ chains on $\operatorname{Ir}(001)$. a $\mathrm{A}(3 \times 1)$ structural unit cell is observed with a non-magnetic $W$ tip (scale bar: $1 \mathrm{~nm}$ ). b With a $\mathrm{Cr}$-coated $\mathrm{W}$ tip the magnetic $(9 \times 2)$ unit cell is resolved. c Line profiles drawn along the stripes at the positions indicated by arrows measured with the W (black) and the Cr-coated (colored) probe tip. Spinresolved line sections differ in periodicity, phase, and amplitude from their spin-averaged counterparts (see main text for details). Scan parameters: a $U=-500 \mathrm{mV}, I=3 \mathrm{nA} ; \mathbf{b} U=100 \mathrm{mV}, I=300 \mathrm{pA}$

assuming an AFM coupling along the chain and a chiral $120^{\circ}$ coupling between adjacent chains. This spin configuration which leads to a $(9 \times 2)$ magnetic unit cell is schematically sketched in Fig. 3a. As mentioned above the AFM Mn-Mn intra-chain coupling can directly be concluded from the doubling of the periodicity in SP-STM as compared to spin-averaged data $\left(a_{\mathrm{Ir}}\right)$ (cf. Fig. 2c). To also explain the corrugation amplitudes and their phase we need to consider that the magnetic corrugation in SPSTM, $\Delta z_{\mathrm{SP}}$, depends on the cosine of the angle $\theta$ included between the magnetization directions of the tip and the sample,

$$
\Delta z_{\mathrm{SP}} \propto P_{\mathrm{t}} \cdot P_{\mathrm{s}} \cdot \cos \theta
$$

with $P_{\mathrm{t}}$ and $P_{\mathrm{s}}$ being the spin polarization of tip and sample, respectively. The expected magnetic contrast can be deduced from the scheme in Fig. 3b. It represents three sample magnetization directions which are rotated by $120^{\circ}$ to another as symbolized by colored arrows. According to Eq. (1), $\Delta z_{\mathrm{SP}}$ is given by the projection of the sample magnetization onto the tip magnetization. Therefore, the maximum $\Delta z_{\mathrm{SP}}$ is expected for a sample magnetization which is almost collinear to the tip magnetization (represented by the black arrow). As symbolized by the lightly colored triangle this condition is fulfilled for the blue arrow in 

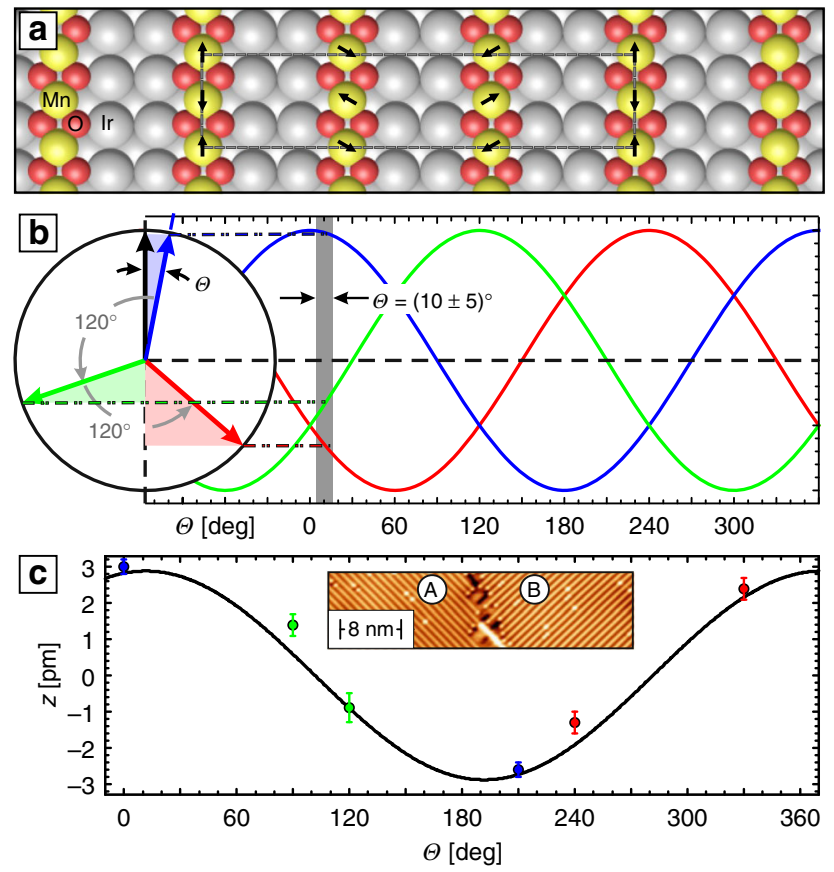

Fig. 3 Interpretation of SP-STM results by a chiral inter-chain exchange. a Schematic model of the chiral $(9 \times 2)$ spin structure of $\mathrm{MnO}_{2} / \operatorname{lr}(001)$. b Sketch of the SP-STM signal expected on a sample with three domains rotated by $120^{\circ}$ to another (see text and Eq. 1 for details). The corrugations data of Fig. $2 \mathrm{~b}$ are best fit with an angle $\theta=(10 \pm 5)^{\circ}$ between tip magnetization (black) and the nearest domain (blue). c Corrugation values determined by fitting line profiles measured on two adjacent domains $A$ and $B$ (inset) as measured with an in-plane tip. The error bar represents the residual sum of squares. The black curve represents the cosine expected for an in-plane rotating spin spiral

Fig. 3b (offset by angle $\theta$ ). In this situation it is unavoidable that the projection of the other two arrows points into the direction opposite to the black arrow. This can also be verified by inspecting the right part of Fig. $3 \mathrm{~b}$, where we plot three cosine functions shifted by $120^{\circ}$.

In other words, from the fundamental principles of SP-STM it follows that (i) whenever we obtain a large contrast on one AFM spin chain the other two chains with spin quantization axes rotated by $\pm 120^{\circ}$ must exhibit a magnetic corrugation which is phase-shifted with respect to the high-contrast row. Furthermore, (ii) even if $\theta$ is relatively small, one of the $\pm 120^{\circ}$-rows exhibits a much lower magnetic contrast since the $\cos \theta$ term in Eq. (1) is close to zero. As marked by a gray box in Fig. 3b, the corrugations measured in Fig. $2 \mathrm{~b}$ can nicely be explained by a tip which is rotated by $\theta=(10 \pm 5)^{\circ}$ with respect to the (blue) domain. As discussed in detail in the Supplementary Note 3 we have performed various SP-STM measurement to identify the spin orientation of the $\mathrm{MnO}_{2}$ chains. Figure 3c shows the magnetic corrugation measured on the three $\mathrm{MnO}_{2}$ chains (blue, red, and green) of two domains (A and B; see inset), respectively, which are rotated by $90^{\circ}$ with respect to another. The black line is the corrugation expected for a spin spiral rotating in the surface plane. The very good agreement with our experimental data suggests an inter-chain coupling characterized by an in-plane $120^{\circ}$ rotation of the azimuthal spin orientation. In order to verify if this spin order is indeed chiral we determined the rotational sense of seven separate $\mathrm{MnO}_{2}$ domains (see Supplementary Note 4). Indeed, our SP-STM measurements show that all domains exhibit the same rotational sense, a result which is highly unlikely under non-chiral conditions $(<2 \%)$.
Density-functional theory calculations. To obtain some insights into the origin of the observed magnetic structures we performed DFT calculations (see Supplementary Note 5 for details). The preferred magnetic ordering along the chains was found to be AFM, in agreement with earlier calculations ${ }^{17}$ and our experimental results (see Fig. 2b). Whereas a weak AFM inter-chain coupling of $0.4 \mathrm{meV}$ per $\mathrm{Mn}$ atom was found previously ${ }^{17}$, our calculations performed at a much denser k-point sampling (using a $24 \times 36$ Monkhorst-Pack grid) leads to a weak FM coupling of $1.7 \mathrm{meV}$. We calculated flat spin spirals with various wave vectors $q$, where the FM (AFM) state corresponds to $q=0(q=0.5)$ in units of $2 / 3 a_{\mathrm{Ir}}$. From our spin spiral calculations, Fig. 4 , we can see that symmetric (Heisenberg-type; blue) exchange interactions lead to a flat dispersion, without any minimum at finite $q$. Our results show that the DMI is largest for a spin spiral with $q=1 / 3$, i.e., the modulation vector found experimentally, lowering the total energy by about 0.3 $\mathrm{meV}$. We have to note, however, that the theoretically obtained Dzyaloshinskii vector, $\mathbf{D}$, points along the chain direction, whereas experiments suggest an in-plane spin spiral, corresponding to $\mathbf{D}$ along the surface normal. To explain the experimentally observed unique rotation sense, there must be a significant out-of-plane component of $\mathbf{D}$, e.g., due to a structural distortion that removes the [110] mirror plane. A similar mechanism has recently been shown to exist for zigzag $\mathrm{Co} / \operatorname{Ir}(001)^{13}$. Indeed, some hints of a potential distortion of the $(3 \times 1)$ structural unit cell can not only be recognized in our data (Fig. 2a), but also in the data published by Ferstl and co-workers (see Figs. 1c, S2 in ref. ${ }^{17}$, and Supplementary Note 6 of this article). In either case atomic resolution data recorded on magnetic TMO chains with non-magnetic tips show some oblique distortion of the expected rectangular surface unit cell. Although this does not directly lead to a non-vanishing effective perpendicular $\mathbf{D}$, it indicates that some structural details or relaxation effects due to the finite size of structural domain still need to be resolved (see Supplementary Note 5).

\section{Discussion}

Recent research indicates that spin-orbit coupling supports an effective spin transfer torque ${ }^{19-21}$ which is particularly important in applications. Different mechanisms have been proposed to explain the relatively low current thresholds necessary to drive skyrmions or chiral domain walls, including inhomogeneous spin currents $^{19}$, Rashba fields, the spin Hall effect ${ }^{20}$, the DMI, or a combination of the latter two ${ }^{21}$. We speculate that a DME-RKKY interaction-induced indirect chiral magnetic exchange may also lead to an extreme reduction of the required current density in layered magnetic structures. It remains to be investigated whether a chiral magnetic interlayer coupling as it has been observed in Dy/Y superlattices ${ }^{11,12}$ can also be found in other material combinations with strongly spin-orbit coupled non-magnetic spacer layers. In more general terms, DME-RKKY interaction may give rise to rather exotic phenomena, such as chiral spinliquid states in spin ice systems ${ }^{22,23}$ or the emergence of new quasiparticles due to the trapping of single electrons in selfinduced skyrmion spin textures ${ }^{24}$.

In summary, we have investigated the intra-chain and interchain magnetic coupling of the quasi one-dimensional system of structurally $(3 \times 1)$-ordered $\mathrm{MnO}_{2}$ on $\operatorname{Ir}(001)$ by spin-polarized scanning tunneling microscopy, angle-resolved photoemission, and density functional theory. Both experimental methods confirm an antiferromagnetic order along the chains, as predicted earlier ${ }^{17}$. In addition, spin-polarized scanning tunneling microscopy reveals a chiral $120^{\circ}$ rotation between adjacent $\mathrm{MnO}_{2}$ chains, resulting in a $(9 \times 2)$ magnetic unit cell. Density functional theory finds that a Dzyaloshinskii-Moriya type enhancement of the RKKY interaction indeed leads to chiral interchain coupling 


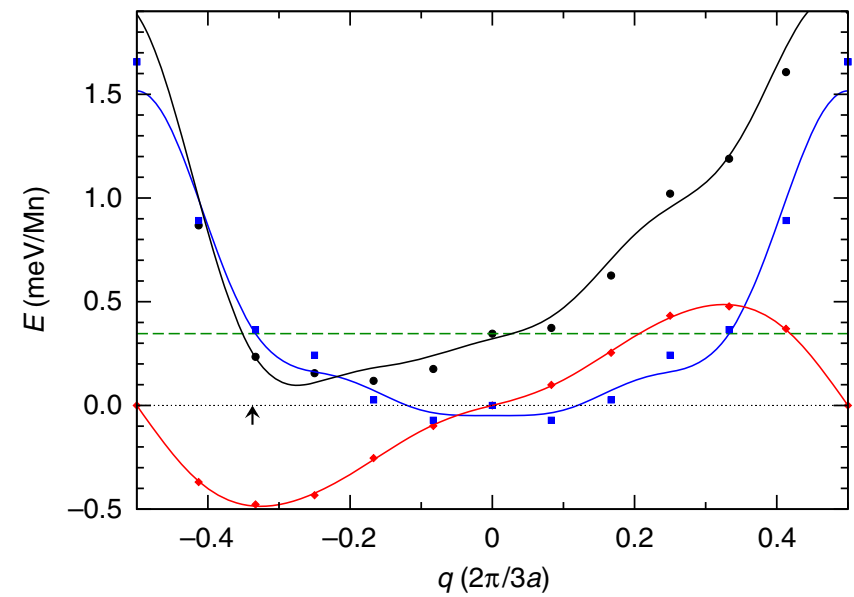

Fig. 4 Results of DFT calculations. Total energy of AFM ordered $\mathrm{MnO}_{2}$ chains on $\operatorname{Ir}(001)$ where the spin directions from chain to chain rotate by an angle of $3 a_{1 r}$. The spins form a cycloidal spin spiral propagating perpendicular to the chains with the rotation axis parallel to the chain direction. The contribution from symmetric exchange interaction is shown by blue squares, from the DMI by red diamonds, and from the magnetocrystalline anisotropy by the dashed line. The sum of these terms is marked by black circles. The blue line is a fit of the symmetric exchange interaction with $\cos (2 n q)$, likewise the DMI was fitted (red line) with $\sin (2 n q)$, both up to $n=4$

with a periodicity in agreement with experiment. However, the orientation of the Dzyaloshinskii vector $\mathbf{D}$ remains to be clarified. Whereas experimental results suggest a perpendicular $\mathbf{D}$, theory predicts a $\mathbf{D}$ vector oriented along the chains.

\section{Methods}

Sample preparation. Sample preparation procedures closely follow published recipes $^{17}$. Initial $\operatorname{Ir}(001)$ preparation comprises cycles of ion-sputtering $\left(1 \mathrm{keV}, \mathrm{Ar}^{+}\right.$, $\approx 2 \mu \mathrm{A}$ ) followed by annealing to $1400 \mathrm{~K}$ in an oxygen atmosphere. The pressure gauge indicates a background pressure $p_{\mathrm{O}_{2}} \approx 1 \times 10^{-8} \mathrm{mbar}$, but since the gas nozzle is located a few $\mathrm{cm}$ above the sample the local oxygen pressure is assumed to be about two orders of magnitude higher. We obtain the $(5 \times 1)$ reconstruction characteristic for clean $\operatorname{Ir}(100)$ by an annealing cycle without oxygen ${ }^{25}$. Oxidizing this surface again in $p_{\mathrm{O}_{2}} \approx 1 \times 10^{-8} \mathrm{mbar}$ at $T_{\mathrm{S}} \approx 850 \mathrm{~K}$ leads to the oxygenterminated $\operatorname{Ir}(100)-(2 \times 1)$ reconstruction ${ }^{17,26,27}$. It served as a substrate for the deposition of 0.33 monolayers (ML) of $\mathrm{Mn}$ at room temperature, followed by final annealing $\left(T_{\mathrm{S}} \approx 1050 \mathrm{~K}\right)$ under oxygen atmosphere.

Scanning tunneling microscopy (STM). STM experiments were performed in a two-chamber ultra-high vacuum (UHV) system (base pressure $p \leq 5 \times 10^{-11} \mathrm{mbar}$ ) equipped with a home-built low-temperature scanning tunneling microscope (LTSTM) (operation temperature $T=5.5 \mathrm{~K}$ ). We used electro-chemically etched polycrystalline $\mathrm{W}$ tips which were flashed by electron bombardment and coated with Fe or Cr for SP-STM measurements ${ }^{28}$.

Angular-resolved photoemission spectroscopy (ARPES). ARPES data were acquired at 130 and $150 \mathrm{eV}$ photon energy at the VUV photoemission beamline (Elettra, Trieste). These photon energies are close to the Cooper minimum of the Ir $5 d$ photoemission (PE) cross section ${ }^{29}$ and enhances the PE signal of Mn $3 d$ states (chains) with respect to the overlapping Ir $5 d$ states (substrate). The spot of the synchrotron light on the sample $(500 \mu \mathrm{m} \times 200 \mu \mathrm{m})$ is much larger than the typical size of domains with parallel $\mathrm{MnO}_{2}$ chains. Thus, ARPES provides a spaceaveraged signal over the two perpendicular orientations of the $\mathrm{MnO}_{2}$ chains. The energy and momentum resolutions were set to $15 \mathrm{meV}$ and $0.02 \AA^{-1}$, respectively.

DFT calculations. Non-collinear DFT calculations were performed using the fullpotential linearized augmented plane wave method as implemented in the Fleur $\operatorname{code}^{30}$. We set up a seven layer film in a $(3 \times 2)$ unit cell as described in ref. ${ }^{17}$, using the local density approximation ${ }^{31}$ with Hubbard $U$ corrections $^{32}$ on the Mn $d$ states $(U=2.7 \mathrm{eV}, J=1.2 \mathrm{eV})$. We confirmed that these values put the Mn $d$ states about $2.2 \mathrm{eV}$ below the Fermi level, in good agreement with the ARPES data presented in Fig. 1d. We used the generalized Bloch theorem to calculate the spin spiral structures and included spin-orbit coupling in first order perturbation theory to estimate the strength of the DMI in this system ${ }^{33}$.

\section{Data availability}

The data that support these findings of this study are available on request from M.S. (STM), P.M. (ARPES), and G.B. (theory).

Received: 19 September 2018 Accepted: 16 May 2019

Published online: 13 June 2019

\section{References}

1. Ruderman, M. A. \& Kittel, C. Indirect exchange coupling of nuclear magnetic moments by conduction electrons. Phys. Rev. 96, 99-102 (1954).

2. Kasuya, T. A theory of metallic ferro- and antiferromagnetism on Zener's model. Prog. Theor. Phys. 16, 45-57 (1956).

3. Roth, L. M., Zeiger, H. J. \& Kaplan, T. A. Generalization of the RudermanKittel-Kasuya-Yosida interaction for nonspherical fermi surfaces. Phys. Rev. 149, 519-525 (1966).

4. Bruno, P. \& Chappert, C. Ruderman-Kittel theory of oscillatory interlayer exchange coupling. Phys. Rev. B 46, 261-270 (1992).

5. Stiles, M. D. Interlayer exchange coupling. J. Magn. Magn. Mater. 200, 322-337 (1999).

6. Hartmann, U. (ed.) Magnetic Multilayers and Giant MagnetoresistanceFundamentals and Industrial Applications (Springer, New York, 2004).

7. Dzialoshinskii, I. E. Thermodynamical theory of 'weak' ferromagnetism in antiferromagnetic substances. J. Exp. Theor. Phys. 5, 1259-1272 (1957).

8. Moriya, T. Anisotropic superexchange interaction and weak ferromagnetism. Phys. Rev. 120, 91-98 (1960).

9. Smith, D. A. New mechanisms for magnetic anisotropy in localised s-state moment materials. J. Magn. Magn. Mater. 1, 214-225 (1976).

10. Fert, A. \& Levy, P. M. Role of anisotropic exchange interactions in determining the properties of spin-glasses. Phys. Rev. Lett. 44, 1538-1541 (1980).

11. Grigoriev, S. V., Chetverikov, Y. O., Lott, D. \& Schreyer, A. Field induced chirality in the helix structure of Dy/Y multilayer films and experimental evidence for Dzyaloshinskii-Moriya interaction on the interfaces. Phys. Rev. Lett. 100, 197203 (2008).

12. Grigoriev, S. V. et al. Interplay of RKKY, Zeeman, and Dzyaloshinskii-Moriya interactions and the nonzero average spin chirality in Dy/Y multilayer structures. Phys. Rev. B 82, 195432 (2010).

13. Dupé, B. et al. Giant magnetization canting due to symmetry breaking in zigzag Co chains on $\operatorname{Ir}(001)$. New J. Phys. 17, 023014 (2015).

14. Khajetoorians, A. A. et al. Tailoring the chiral magnetic interaction between two individual atoms. Nat. Commun. 7, 10620 (2016).

15. Bouaziz, J. et al. Chiral magnetism of magnetic adatoms generated by Rashba electrons. New J. Phys. 19, 023010 (2017).

16. Hermenau, J. et al. A gateway towards non-collinear spin processing using three-atom magnets with strong substrate coupling. Nat. Commun. 8, 642 (2017).

17. Ferstl, P. et al. Self-organized growth, structure, and magnetism of monatomic transition-metal oxide chains. Phys. Rev. Lett. 117, 046101 (2016).

18. Kubetzka, A. et al. Revealing antiferromagnetic order of the Fe monolayer on $\mathrm{W}(001)$ : spin-polarized scanning tunneling microscopy and first-principles calculations. Phys. Rev. Lett. 94, 087204 (2005).

19. Jonietz, F. et al. Spin transfer torques in $\mathrm{MnSi}$ at ultralow current densities. Science 330, 1648-1651 (2010).

20. Emori, S., Bauer, U., Ahn, S.-Mi, Martinez, E. \& Beach, G. S. D. Currentdriven dynamics of chiral ferromagnetic domain walls. Nat. Mater. 12, 611-616 (2013)

21. Ryu, K.-S., Yang, S.-H., Thomas, L. \& Parkin, S. S. P. Chiral spin torque arising from proximity-induced magnetization. Nat. Commun. 5, 3910 (2014).

22. Machida, Y., Nakatsuji, S., Onoda, S., Tayama, T. \& Sakakibara, T. Timereversal symmetry breaking and spontaneous Hall effect without magnetic dipole order. Nature 463, 210-213 (2009).

23. Flint, R. \& Senthil, T. Chiral RKKY interaction in Pr2Ir2O7. Phys. Rev. B 87, 125147 (2013).

24. Luis, B. Magnetic skyrmionic polarons. Nano Lett. 17, 7358-7363 (2017).

25. Schmidt, A., Meier, W., Hammer, L. \& Heinz, K. Deep-going reconstruction of $\operatorname{Ir}(100)-5 \times 1$. J. Phys. Condens. Matter 14, 12353-12365 (2002).

26. Rhodin, T. N. \& Broden, G. Preparation and chemisorptive properties of the clean normal and reconstructed surfaces of $\operatorname{Ir}(100) \mid$ role of multiplets. Surf. Sci. 60, 466-484 (1976). 
27. Johnson, K., Ge, Q., Titmuss, S. \& King, D. A. Unusual bridged site for adsorbed oxygen adatoms: Theory and experiment for Ir100- $(1 \times 2) \mathrm{O}$. J. Chem. Phys. 112, 10460-10466 (2000).

28. Bode, M. Spin-polarized scanning tunnelling microscopy. Rep. Prog. Phys. 66, 523-583 (2003).

29. Yeh, J. J. \& Lindau, I. Atomic subshell photoionization cross sections and asymmetry parameters: $1 \leq Z \leq 103$. At. Data Nucl. Data Tables 32, 1-155 (1985).

30. Kurz, Ph, Förster, F., Nordström, L., Bihlmayer, G. \& Blügel, S. Ab initio treatment of non-collinear magnets with the full-potential linearized augmented planewave method. Phys. Rev. B 69, 024415 (2004).

31. Vosko, S. H., Nusair, M. \& Wilk, L. Accurate spin-dependent electron liquid correlation energies for local spin density calculations: a critical analysis. Can. J. Phys. 58, 1200-1211 (1980).

32. Shick, A. B., Lichtenstein, A. I. \& Pickett, W. E. Implementation of the LDA + $\mathrm{U}$ method using the full-potential linearized augmented plane-wave basis. Phys. Rev. B 60, 10763-10769 (1999).

33. Heide, M., Bihlmayer, G. \& Blügel, S. Describing Dzyaloshinskii-Moriya spirals from first principles. Phys. B 404, 2678-2683 (2009).

\section{Acknowledgements}

Experimental work was supported by DFG through FOR 1700 (project E6), SPP 2137 "Skyrmionics" (BO 1468/26-1), and by the Dresden-Würzburg Center for Topological Quantum Matter Research (ct.qmat). A.K.K. acknowledges receipt of a fellowship from the ICTP-TRIL Programme, Trieste, Italy.

\section{Author contributions}

M.S., R.C., M.V. and J.K. performed and analyzed STM measurements. P.M., P.M.S.,

A.K.K. and C.C. performed ARPES experiments and analyzed the data. G.B., A.B. and S.

B. provided the theoretical framework. All authors discussed the results and contributed in writing the manuscript.

\section{Additional information}

Supplementary Information accompanies this paper at https://doi.org/10.1038/s41467019-10515-3.

Competing interests: The authors declare no competing interests.

Reprints and permission information is available online at http://npg.nature.com/ reprintsandpermissions/

Journal peer review information: Nature Communications thanks the anonymous reviewers for their contribution to the peer review of this work.

Publisher's note: Springer Nature remains neutral with regard to jurisdictional claims in published maps and institutional affiliations.

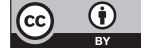

Open Access This article is licensed under a Creative Commons Attribution 4.0 International License, which permits use, sharing, adaptation, distribution and reproduction in any medium or format, as long as you give appropriate credit to the original author(s) and the source, provide a link to the Creative Commons license, and indicate if changes were made. The images or other third party material in this article are included in the article's Creative Commons license, unless indicated otherwise in a credit line to the material. If material is not included in the article's Creative Commons license and your intended use is not permitted by statutory regulation or exceeds the permitted use, you will need to obtain permission directly from the copyright holder. To view a copy of this license, visit http://creativecommons.org/ licenses/by/4.0/.

(C) The Author(s) 2019 